\title{
Quantitative aspects of the excretion of radioactive and microbiologically active material in urine after parenteral hydroxocobalamin
}

\author{
J. F. ADAMS AND ELIZABETH H. KENNEDY
}

From the Southern General Hospital, Glasgow

SYNOPSIS The radioactive material in urine after parenteral radioactive hydroxocobalamin is composed of radioactive cyanocobalamin, radioactive hydroxocobalamin, and a radioactive anionic complex. Studies in vitro suggest that these materials have a similar microbiological activity. Comparison of the amounts of radioactive and microbiologically active material in urine after parenteral radioactive hydroxocobalamin show a significant correlation both in the first and second 24 hours after injection showing that the amount of radioactivity is an acceptable measure of the loss of cobalamin in urine. The slope of the regression lines obtained from cobalamin-deficient and normal subjects implies that there is no equilibration of injected radioactive hydroxocobalamin and body stores: this may be due to the absence of hydroxocobalamin from tissues or to the fact that tissue hydroxocobalamin is bound and incapable of equilibration.

When radioactive cyanocobalamin is injected the radioactive material subsequently excreted in urine is in the form of intact molecules of radioactive cyanocobalamin (Smith, 1952, 1953; MacLean and Bloch, 1954). There is a significant correlation between the amount of radioactivity excreted, expressed as a percentage of the dose injected, and the amount of microbiologically active material excreted (Adams, 1961). These facts justify the use of radiometric methods for measurement of urinary excretion of radioactive cyanocobalamin after parenteral injection.

The position is different with regard to radioactive hydroxocobalamin. After parenteral injection the radioactive material excreted in the following 24 hours in urine is not radioactive hydroxocobalamin but is composed of radioactive hydroxocobalamin, radioactive cyanocobalamin, and a radioactive anionic complex (Kennedy and Adams, 1965). Therefore, before accepting radiometric values as valid measurements of the urinary loss of radioactive hydroxocobalamin, it is essential that the relative microbiological activities of these complexes be established and that inquiry be made about the relationship between the amount of radioactivity excreted and the amount of microbiologically active material excreted.

Received for publication 10 June 1965.
We report here observations on the microbiological activity of the complexes and on the relationship between radioactivity and microbiologically active material excreted after parenteral radioactive hydroxocobalamin in normal and cobalamin-deficient subjects.

\section{MATERIALS, METHODS, AND RESULTS}

STUDIES IN VITRo Three separate procedures were undertaken.

1 The microbiological activities of solutions of pure \& cyanocobalamin and pure hydroxocobalamin, as judged by visual localization on column chromatography, were $\bigcirc$ compared in a conventional assay procedure (methods of Ross, 1952; Hutner, Bach, and Ross, 1956). Two sets $\frac{7}{0}$ of solutions, each in duplicate, were set up, the first containing cyanocobalamin in concentrations of $1 \cdot 25, \mathrm{~N}$ $2 \cdot 5,5,10,15,25$, and $50 \mu \mu \mathrm{g} . / \mathrm{ml}$., the second containing hydroxocobalamin in the same concentrations. The 0 activity of the two compounds was found to be the same at similar concentrations as judged by the optical densities after inoculation and incubation for five days.

From this experiment, which has been repeated with $\stackrel{0}{\leftarrow}$ similar results on several occasions, we conclude that $\mathscr{\mathscr { S }}$ cyanocobalamin and hydroxocobalamin have an exual microbiological activity at similar concentrations at least for Euglena gracilis z strain.

$2{ }^{57} \mathrm{Co}$ hydroxocobalamin, $500 \mu \mathrm{g}$., was added to a $1,000 \mathrm{ml}$. pooled urine collection. After incubation for three hours qualitative analysis by the method of Kennedy 
and Adams (1965) showed that only $59 \%$ of the radioactive material was in the form of hydroxocobalamin, the remainder being cyanocobalamin $(14 \%)$ and anionic complex $(20 \%)$. An aliquot of the solution was diluted with cobalamin-free water and assayed on three occasions at a suitable dilution against standards of pure cyanocobalamin. The calculated mean value of the original sample was equivalent to cyanocobalamin in a concentration of $0.5 \mu \mathrm{g} . / \mathrm{ml}$.

3 A sample of ${ }^{57} \mathrm{Co}$ hydroxocobalamin solution, $0.01 \mu \mathrm{g}$. $0.10 \mu \mathrm{c}$. $/ \mathrm{ml}$., was found on qualitative analysis to have undergone spontaneous degradation, the mixture containing only $63 \%$ hydroxocobalamin, the remainder being cyanocobalamin $(3.5 \%)$ and anionic complex $(30 \%)$. The solution was diluted with cobalamin-free water and assayed at a suitable dilution on three occasions. The mean value was equivalent to cyanocobalamin in a concentration of $0.009 \mu \mathrm{g} . / \mathrm{ml}$.

From these two observations we conclude that the anionic complex has a microbiological activity equal to that of cyanocobalamin and hydroxocobalamin.

STUDIES IN vivo Two groups of patients were studiedThe first group, 20 in number, were ward in-patients with a variety of diseases none of which are known to be associated with deranged cobalamin metabolism. All had normal serum vitamin $B_{12}$ levels. After a collection of urine each patient was given a single intramuscular injection of $1,000 \mu \mathrm{g} .0 \cdot 1 \mu \mathrm{c} .{ }^{57} \mathrm{Co}$ hydroxocobalamin and all urine passed in the subsequent 72 hours was collected in 24-hour collection periods. The second group was composed of three patients who had a macrocytic anaemia, megaloblastic marrow, histamine-fast achlorhydria, and low serum vitamin $B_{12}$ level on admission and who, at a later date, were found to have a failure of absorption of orally administered radioactive vitamin $B_{12}$, corrected by the addition of intrinsic factor, as judged by the Schilling test. After a urine collection one patient received a single injection of ${ }^{57} \mathrm{Co}$ hydroxocobalamin, $1,000 \mu \mathrm{g} ., 0 \cdot 1 \mu \mathrm{c}$, and the urine passed in the following 72 hours was collected in 24-hour collection periods. The other two patients were treated with injections of $1,000 \mu \mathrm{g} ., 0 \cdot 1 \mu \mathrm{c} .{ }^{57} \mathrm{Co}$ hydroxocobalamin given at 72 -hour intervals, one patient receiving 10 injections and the other 12 injections. Urine collections were made for 24-hour periods from the time of the first injection until 72 hours after the last injection. The isotope, microbiological assay, and other technical methods have already been described (Adams, 1961; Kennedy and Adams, 1965).

The amounts of radioactivity and microbiologically active material excreted by both groups of patients in 24 hours after injection were always considerable and are shown in Figure 1. Much less was excreted in the second 24 hours after injection and in some cases the amounts were insignificant. The significant values are recorded in Figure 2. In the third 24-hour collections very little was excreted, particularly by the normals, and the number of results available was inadequate for analysis. The essential statistical values and analysis of the results for the first and second 24-hour periods are set out in Table I.

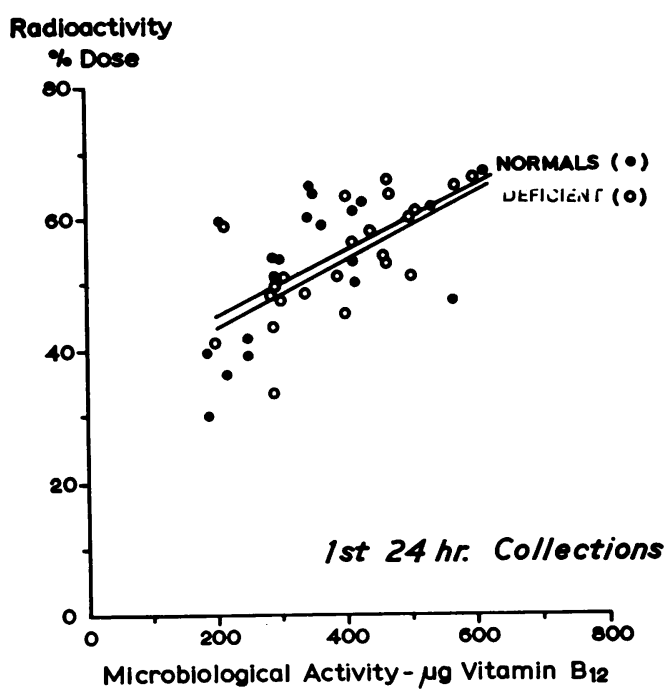

FIG. 1.

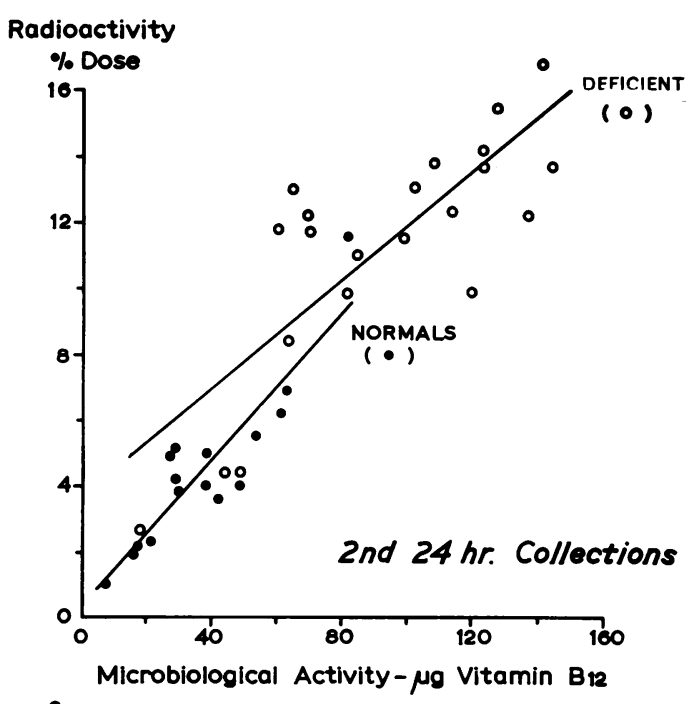

FIG. 2.

FIGS. 1 and 2. Correlation of radioactivity and microbiological activity in urines in the first and second 24 hours after parenteral ${ }^{\circ} \mathrm{C}$ Co hydroxocobalamin in normal and initially $B_{12}$-deficient subjects.

\section{DISCUSSION}

The results of the studies in vitro infer that cyanocobalamin, hydroxocobalamin, and the anionic complex have equal microbiological activities. We have not yet been able to isolate the anionic complex in pure form in known concentration and to compare it directly with cyanocobalamin and hydroxocobalamin in an assay but the results of the studies 
TABLE I

ESSENTIAL STATISTICAL RESULTS OBTAINED IN THE INVESTIGATION

\begin{tabular}{|c|c|c|c|c|c|c|}
\hline \multirow[b]{2}{*}{ Period (hr.) } & \multirow[b]{2}{*}{ Patients } & \multirow[b]{2}{*}{ No. of Values } & \multicolumn{2}{|l|}{ Mean Values } & \multirow[b]{2}{*}{$\begin{array}{l}\text { Coefficient of } \\
\text { Correlation }(r)\end{array}$} & \multirow[b]{2}{*}{$\begin{array}{l}\text { Regression of Assay ( } x \\
\text { on Isotope }(y)\end{array}$} \\
\hline & & & $\begin{array}{l}\text { Microbiological } \\
\text { Activity }\left(\mu g . B_{12}\right)\end{array}$ & $\begin{array}{l}\text { Radioactivity } \\
(\% \text { Dose })\end{array}$ & & \\
\hline \multirow{2}{*}{$0-24$ hours } & Deficient & 23 & $394 \cdot 65$ & 53.66 & 0.69 & $Y=32.28+0.05418 x$ \\
\hline & Normal & 20 & $347 \cdot 90$ & $52 \cdot 91$ & 0.58 & $Y=35.38+0.05038 x$ \\
\hline \multirow{2}{*}{ 24-48 hours } & Deficient & 21 & $92 \cdot 76$ & $12 \cdot 24$ & $0 \cdot 79$ & $Y=3.67+0.08155 x$ \\
\hline & Normal & 16 & $37 \cdot 62$ & $4 \cdot 50$ & 0.81 & $Y=0.28+0.1122 x$ \\
\hline
\end{tabular}

in vitro constitute good evidence that the three compounds are equally active. This finding makes it permissible to compare the amounts of radioactive and microbiologically active material present in urine after parenteral hydroxocobalamin. The significant correlation between these values, both in the first and second 24 hours after injection, can therefore be taken as evidence that the amount of radioactivity excreted is a true measure of the urinary loss of cobalamins. As there is evidence that the partial conversion of hydroxocobalamin to cyanocobalamin and anionic complex takes place after excretion of hydroxocobalamin by the nephron (Kennedy and Adams, 1965), the correlation thus means that the amount of radioactivity in urine is an acceptable measure of the loss of hydroxocobalamin in urine following parenteral dosing.

The similarity of the slope of the regression line obtained from the cobalamin-deficient patients and from the normal patients merits comment. This finding was obtained in a previous study using cyanocobalamin and it was concluded that the results were evidence that parenteral cyanocobalamin did not equilibrate with body stores to any significant degree. Had there been any significant degree of equilibration then, according to the isotope dilution principle, the amount of radioactivity would have represented only a fraction of the amount of microbiologically active material. From this it was concluded that cyanocobalamin does not exist as such in the body but in the form which renders it incapable of equilibrating with injected cyanocobalamin (Adams, 1961). Similarly it can be concluded from the results presented here that there is no significant degree of equilibration of injected radioactive hydroxocobalamin and body stores and therefore that hydroxocobalamin does not exist as such in the body. This may mean that hydroxocobalamin is converted to some other complex such as coenzyme $\mathrm{B}_{12}$ or may simply be a reflection of the fact that hydroxocobalamin is bound to protein and that the process of binding renders it incapable of equilibration with free hydroxocobalamin.
Anaemia affects the amount of radioactivity excreted in urine after parenteral hydroxocobalamin, $\infty$ the effect probably resulting from the greater plasma volume and consequently increased plasma- $\infty$ binding capacity (Adams and Kennedy, 1965). The 음 comparable mean values for the two groups of $\rightarrow$ patients in this series does not contradict this $Z$ finding. The increased plasma-binding capacity in the anaemic patients would not obtain after the first injection of hydroxocobalamin as this would saturate plasma binders. Thus only the values for amounts excreted after the first injection in anaemic patients would reflect the difference previously reported between anaemic and non-anaemic subjects, and these values are submerged in the subsequent values obtained from the same patients.

We are grateful to Dr. J. J. F. Merry of Merck, Sharp and Dohme Ltd., for supplies of ${ }^{57} \mathrm{Co}$ hydroxocobalamin, $\overrightarrow{\overrightarrow{0}}$ and to Professor T. Ferguson Rodger for laboratory facilities. We acknowledge with thanks a grant from the Secretary of State for Scotland on the advice of the Advisory Committee on Medical Research.

\section{REFERENCES}

Adams, J. F. (1961). The urinary excretion of assayable vitamin $\mathbf{B}_{12}$ and radioactivity after parenteral ${ }^{58} \mathrm{Co} \mathrm{B}_{12}$ in man.J. clin. Path., $14,351$.

and Kennedy, E. H. (1965). Hydroxocobalamin: Excretion and retention after parenteral doses in anemic and nonanemic $D$ subjects, with reference to the treatment of vitamin $B_{12}$ 윽 deficiency states. J. Lab. clin. Med., 65, 450.

Hutner, S. H., Bach, M. K., and Ross, G. I. M. (1956). A sugar- N containing basal medium for vitamin $\mathbf{B}_{12}$-assay with Euglena, application to body fluids. J. Protozool., 3, 101.

Kennedy, E. H., and Adams, F. J. (1965). The nature of the radioactive material in urine after parenteral ${ }^{57} \mathrm{Co}$ hydroxocobalamin $\omega$ in man. Clin. Sci., in the press.

MacLean, L. D., and Bloch, H. S. (1954). Gastrointestinal absorption and urinary excretion of vitamin $\mathrm{B}_{12}-\mathrm{Co}^{60}$. Proc. Soc. exp. Biol. (N.Y.), 87, 171 .

Ross, G. I. M. (1952). Vitamin $B_{12}$ assay on body fluids using Euglena $\mathscr{D}$ gracilis. J. clin. Path., 5, 250.

Smith, E. L. (1952). Radioactive penicillin and vitamin $\mathbf{B}_{12}$. Brit. med. Bull., 8, 203.

(1953). Tracer studies with the vitamins $B_{12}$ inRadioisotope Techniques. Proceedings of the Isotope Techniques Conference, Oxford, July 1951, sponsored by the Atomic Energy Research $\stackrel{(D}{ }$ Establishment, vol. 1, medical and physiological applications, p. 281. H.M.S.O., London. 